\title{
Usefulness of Light Emitting Diode (LED) fluorescent microscopy as a tool for rapid and effective method for the diagnosis of pulmonary tuberculosis
}

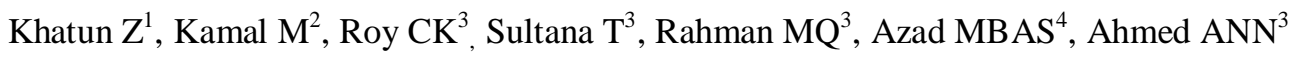 \\ ${ }^{1}$ DGHS, Dhaka, ${ }^{2}$ NTRL, NIDCH, Dhaka, ${ }^{3}$ Department of Clinical Pathology, BSMMU, Dhaka, \\ ${ }^{4}$ Narsingdi Zilla Hospital, Narsingdi
}

\begin{abstract}
Background: Tuberculosis remains world's leading cause of death from a single infectious agent. Fluorescence microscopy offers well-described benefits, comparing with brightfield microscopy, for the evaluation sputum smear samples for tuberculosis. We evaluated the diagnostic performance of fluorescence microscopy, using novel Light Emitting Diode (LED) technology as an alternative to the conventional fluorescence microscopy by Auramine stain as well as brightfield microscopy by ZiehlNeelsen $(\mathrm{ZN})$ stain. Objectives: The objective of the study was to see the usefulness of LED fluorescent microscopy in the diagnosis of pulmonary tuberculosis. Methods: This is a prospective study consisted of 150 sputum samples from the patients of NIDCH, Mohakhali. All samples were stained by auramine and ZN stain at BSMMU and culture was done in Lowenstein-Jensen (L-J) media as gold standard at NTRL, Mohakhali. Results: In this study total 66(44\%) out of 150 sputum specimens were positive for Mycobacterium Tuberculosis by culture. Sensitivity and specificity documented for the different modalities were $95.38 \%$ and $94.11 \%$, respectively, for the LED assessment; $68.18 \%$ and $90.47 \%$, respectively, for the CFM assessment; and $56.06 \%$ and $97.61 \%$, respectively, for brightfield microscopy by $\mathrm{ZN}$ stain. The difference in their case detection rate was statistically significant $\left(\chi^{2}=119.38, \mathrm{p}<0.001\right)$. Conclusion: Fluorescence Microscopy (FM) is more sensitive than $\mathrm{ZN}$ for diagnosis of pulmonary tuberculosis. However, since FM is more sensitive and rapid, using this method (LED) in clinical laboratories with large specimen numbers is recommended.
\end{abstract}

\section{Introduction}

Tuberculosis (TB) was declared as a global emergency in 1993. It remains world's leading cause of death from a single infectious agent ${ }^{1}$. It is also a major public health problem in Bangladesh and ranks sixth among the 22 highest burden tuberculosis countries in the world ${ }^{2}$. Nearly $2.14 \%$ of the population become infected every year ${ }^{3}$. 300,000 progress to disease and at least 70,000 people die of TB each year. Incidence and prevalence of tuberculosis in Bangladesh are 225/100,000 per year and 391/100,000 per year respectively ${ }^{4}$. For developing countries with a large number of cases and financial constraints, evaluation of rapid and less expensive diagnostic methods like demonstration of acid-fast bacilli (AFB) in smears has great importance ${ }^{5}$.

Direct microscopy for AFB is widely used method for diagnosis and confirmation of pulmonary tuberculosis and when positive, defines the more infectious cases ${ }^{6}$. This method is highly specific, faster and cheaper for detection of AFB in sputum. But, there are several drawbacks of this method. First of all, ZN stain has low sensitivity relative to fluorescent stain $^{7}$ and culture ${ }^{8}$. Secondly, it takes more time to scan-at least 300 field. Thirdly, it needs experienced pathologist. Finally, it often miss the paucibacilary tuberclosis and when the patient is co-infected with HIV. Technical error is also more common in case of $\mathrm{ZN}$ stain, in which heated carbol fuchsin is very much important ${ }^{9}$.

Last of all, the new light emitting diode (LED) fluorescent microscope is cheaper and with more life expectancy than Conventional fluorescent microscope (CFM). LEDs excite auramine without producing UV light ${ }^{10}$. It also produce minimal heat and contain no hazardous materials. Also there is no need of dark room for LED fluorescent microscope like others. Moreover, as LED needs low power consumption, it can be operated by portable battery ${ }^{11}$. It gives us the opportunity to diagnose TB easily at earliest time with more comprehension.

In contrast to common perception, the laboratory costs of performing Fluorescence Microscopy (FM) are actually cheaper than $\mathrm{ZN}$ in Thailand. Although the initial investment cost for FM is substantially greater than for $\mathrm{ZN}$, this increased cost is offset by a reduction in reagent/ chemical, staff workload and overhead costs ${ }^{11}$. 
If we can spread the applicability of FM, it will minimize the time to detect TB-bacilli, initiation of therapy and decrease the burden of TB in our country. Therefore, it will be generally accepted that the LED fluorescent method should be given preference over the $\mathrm{ZN}$ methods.

\section{Materials and Methods}

This cross sectional study was carried out from October 2009 to September 2010 in the Department of Clinical pathology, Department of Microbiology and Immunology, BSMMU, Dhaka and National Tuberculosis Reference Laboratory (NTRL) of National Institute of Diseases of Chest and Hospital (NIDCH), Mohakhali, Dhaka. The main objective of the study was to compare the findings of Auramine stained sputum smear by LED fluorescent microscopy with conventional fluorescent microscopy and to compare with ZiehlNeelsen (ZN) stained sputum smear by bright field microscopy for the diagnosis of pulmonary tuberculosis.

The validity of different discrimination indices were evaluated by calculating their sensitivity, specificity, accuracy, Positive predictive value and Negative predictive value. Smear reporting is done according to table I.

\section{Results}

Out of total 150 cases it was observed that 66(44\%) cases were diagnosed as pulmonary tuberculosis by culture method as a gold standard. LED showed the nearest result as $67(44.67 \%)$ positive cases, in which 1 case was found as false positive. On the other hand CFM detected 53(35.33\%) cases positive and missed 13 cases (Figure 1).

Only $39(26 \%)$ cases were detected by bright field microscopy with $\mathrm{ZN}$ stain in which 27 cases were missed and detected as false negative. The difference in the case-yields was found to be highly significant $\left(\chi^{2}=119.38, \mathrm{p}<0.001\right)$ (Table II).

Table I: Grading Scales for bright field (Ziehl-Neelsen) and Fluorescence microscopy (WHO,IUATLD, 2007)

\begin{tabular}{|c|c|c|c|}
\hline $\begin{array}{l}\text { Union/ WHO } \\
\text { Scale } \times 1000 \\
\text { field = HPF } \\
\quad \text { Result } \\
\end{array}$ & $\begin{array}{c}\text { Bright fiels } \\
\text { (x1,000 magnification; } \\
1 \text { length }=2 \mathrm{~cm} \\
=100 \mathrm{HPF} \text { ) }\end{array}$ & $\begin{array}{c}\text { Fluorescence } \\
(\times 200-250 \\
\text { magnification; } 1 \text { length } \\
=30 \text { fields }=300 \mathrm{HPF})\end{array}$ & $\begin{array}{c}\text { Fluorescence } \\
\text { (x400 magnification; } \\
1 \text { length=40 fields } \\
=200 \mathrm{HPF} \text { ) }\end{array}$ \\
\hline Negative & Zero AFB/100 HPF & Zero AFB/1 Length & Zero AFB/1 Length \\
\hline Scanty & 1-9 AFB/100 HPF & 1-29 AFB/1 Length & 1-19 AFB/1 Length \\
\hline $1+$ & 10-99 AFB/100 HPF & 30-299 AFB/1 Length & 20-199 AFB/1 Length \\
\hline $2+$ & $\begin{array}{l}\text { 1-10 AFB/1 HPF } \\
\text { (on average } 50 \mathrm{HPF} \text { ) }\end{array}$ & 10-100 AFB/1 field & 5-50 AFB/1 field \\
\hline $3+$ & $\begin{array}{l}>10 \mathrm{AFB} / 1 \mathrm{HPF} \\
\text { (on average } 20 \mathrm{HPF} \text { ) }\end{array}$ & $>100$ AFB/1 field & $>50 \mathrm{AFB} / 1$ field \\
\hline
\end{tabular}

$\mathrm{HPF}=$ high-power field; $\mathrm{AFB}=$ acid-fast bacilli
Table II: Sensitivity, Specificity, Accuracy, Positive and Negative predictive values of sputum examination by ZN stain (Bright field Microscope), Conventional (CFM) and LED fluorescent microscope by auramine stain $(n=150)$.

\begin{tabular}{lccc}
\hline & ZN & CFM & LED \\
\hline Sensitivity & 56.06 & 68.18 & 95.38 \\
Specificity & 97.61 & 90.47 & 94.11 \\
Accuracy & 79.33 & 80.66 & 94.66 \\
PPV & 94.87 & 84.90 & 92.53 \\
NPV & 73.87 & 78.35 & 96.38 \\
\hline
\end{tabular}

(Sensitivity, Specificity, Accuracy, Positive and Negative predictive values are expressed in percentage)

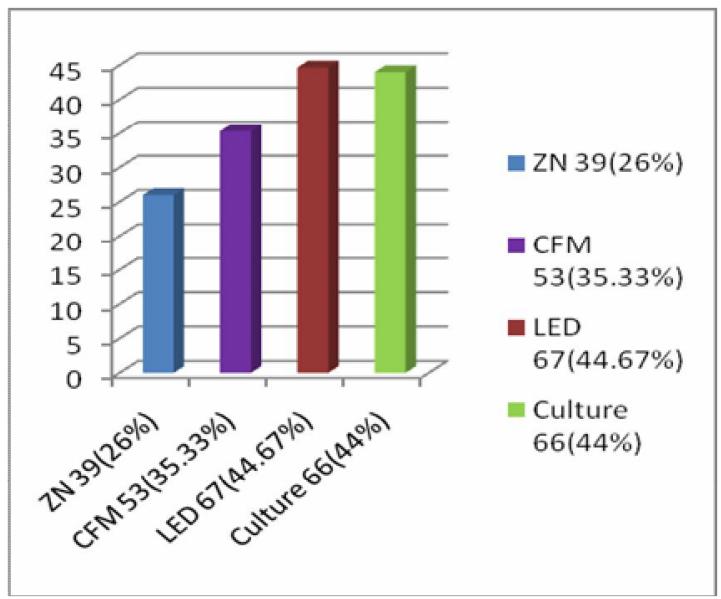

Fig. 1: Bar diagram shows results of Bright field microscopy of sputum by $\mathrm{ZN}$, Conventional (CFM) and LED fluorescent microscopy by Auramine stain and culture for mycobacterium.

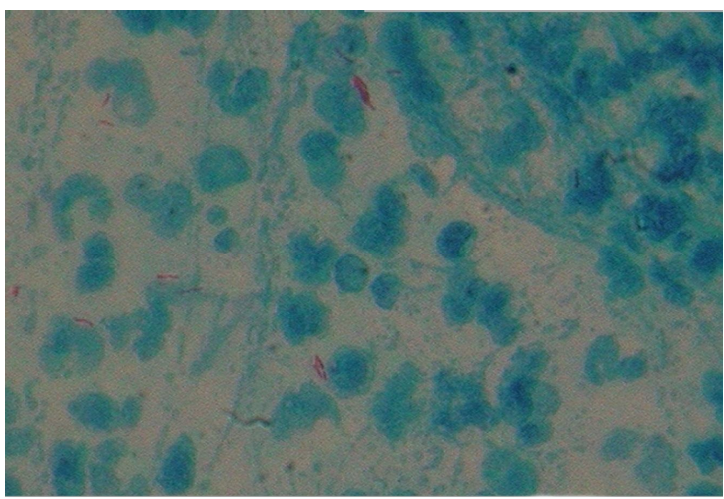

Fig-2: Photomicrograph of Ziehl-Neelsen stain sputum smear showing TB bacilli (AFB +++) (x1000)

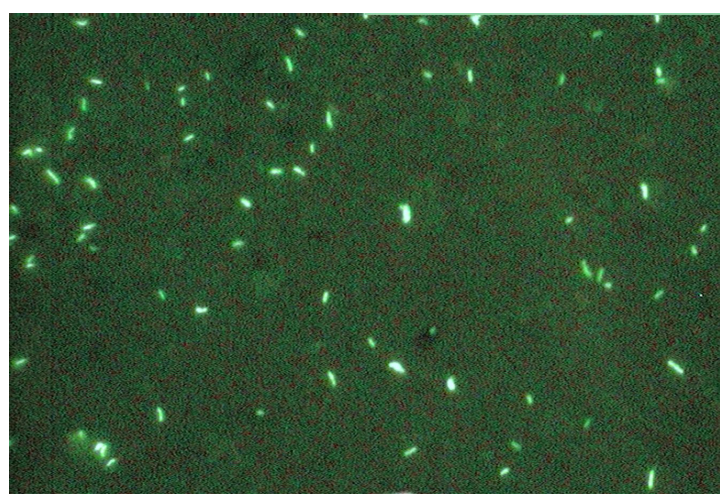

Fig-3: Photomicrograph of Auramine $O$ stain sputum smear showing TB bacilli (AFB +++) by Conventional Fluorescent Microscopy (x200) 


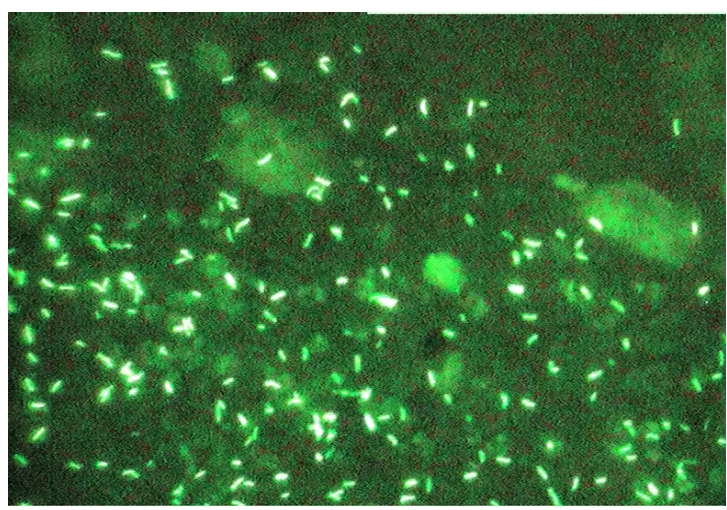

Fig-4: Photomicrograph of Auramine $\mathrm{O}$ stain sputum smear showing TB bacilli (AFB +++) by Light Emitting Diode (LED) Fluorescent Microscopy (x200)

\section{Discussion}

Tuberculosis (TB) is a major public health problem in Bangladesh since long. Estimates suggest that daily about 880 new TB cases and 176 TB deaths occur in the country ${ }^{4}$. Nearly one-third of the global population, i.e. two billion people, is infected with Mycobacterium tuberculosis and thus at risk of developing the disease. More than nine million people develop active TB every year and about two million dies.

Despite all the advances made in the treatment and management, still tuberculosis is a public health problem in Bangladesh with adverse social and economic consequences. Current recommendations for the control of tuberculosis emphasize case detection so as to allow treatment of patients and thereby limit the transmission of the bacilli. The mainstay for its control is the rapid and accurate identification of the infected individuals ${ }^{12}$.

A number of alternative diagnostic tests that use molecular and immunological methods have been developed. While molecular methods overcome the insensitivity of smear method and the time required for culture, they depend upon retrieval of a specimen from the site of infection and require a well-prepared laboratory and well- trained personnel. The simplest rapid method is the detection of acid-fast bacilli by microscopy. In developing countries, microscopy of sputum is by far the fastest, cheapest and more reliable method for diagnosis of pulmonary tuberculosis. The estimated detection limit of microscopy is $10^{4}$ bacilli/ml of sputum ${ }^{13}$.

In this study on evaluation of the microscopic techniques by comparing them with the gold standard culture technique, it was found that in case of $\mathrm{ZN}$ stain there was agreement in $79.33 \%$ cases and disagreement in $20.67 \%$ whereas for Auramine-O(AO) stain there was agreement in
94.67\% cases and disagreement in $5.33 \%$ cases by LED. This proves that AO stain examined by LED is a better method for its close comparability to the gold standard technique. These were almost comparable with other several studies ${ }^{14-18}$.

Sputum culture is widely regarded to be the most sensitive test for the detection of pulmonary TB, but its routine use in resource-limited settings is hampered by excessive cost, slow turnaround times, and the need for adequate laboratory infrastructure. In practice, improvements in direct sputum sample evaluation that result from improved sensitivity and/or improved access to decentralized diagnostic services remain highly relevant.

However, the need for rapid smear results and effective treatment of the most infection TB cases remains paramount. The efficacy of LED fluorescence microscopy proved to be much higher than Conventional fluorescent microscopy and bright field microscopy and comparable to that of culture. In this study, AuramineO (AO) stained sputum smear has been found to improve significantly the sensitivity, predictive value of negative test, percentage of false negative and efficiency. So, LED microscopy of sputum by AO staining can be used effectively along with $\mathrm{ZN}$ stain for the diagnosis of pulmonary tuberculosis instead of doing difficult and time consuming culture method in the peripheral health centre of our country.

\section{Acknowledgement}

I would like to thank all the consultants, junior doctors and staff of the Department of Clinical pathology, BSMMU, Department of Microbiology and Immunology, BSMMU, NTRL, NIDCH, Mohakhali, Dhaka for their sincere help in conducting this study.

\section{Reference}

1. Franco M.D, Salaniponi F, Nayangulu D S. Case finding with a single sputum smear and household bleach. Int J Tuberc Lung Dis 2009; 1(1):144-147

2. WHO, Available from www. who.int/entity/tb/ publications/global-report/2009/pdf/bgd.pdf, 2009;

3. WHO, Bangladesh, TB. Available at: http:// www.whoban.org/communicable dis. tb. Html,Updated 23 March,2006.

4. National guidelines and operational manual for tuberculosis control-Fourth edition,DGHS, WHO country office for Bangladesh 2009:1-14.

5. Laifangbam S,Singh HL. A comparative study of fluorescent microscopy with Ziehl-Neelsen staining and culture for the diagnosis of pulmonary tuberculosis. 
Kathmandu University Medical Journal 2009; 7:226230.

6. Ziaee M, Namaei M, Khazaei M. Comparison of the value of two different sputum staining for diagnosis of acid-fast bacilli. Iranian J Clinical Infectious Diseases 2008; 3(2):99-102.

7. Steingert KR, Henry M,vi vienne. Fluorescence versus conv-entional sputum smear microscopy for tuberculosis, a systematic review. Lancet Infect Dis 2006; 6:570-81.

8. Murray SJ, Barrett A, Magee JG, Freeman R. Optimization of acid fast smears for the direct detection of mycobacteria in clinical samples. J Clin Pathol 2003; $56: 613-15$.

9. Dinnes J, Deeks J, Kunst H, Gibson A, Gummins E, Waugh N, Drobniewski F, Lalvani A. A systematic review of rapid diagnostic tests for the detection of tuberculosis infection. Health Technol Assess 2007; 11(3):1-196

10. Minion J, Sohn H, Pai M. Light Emitting Diode technologies for TB diagnosis: what is the market. Expert Rev. Med. Devices 2009; 6(4):341-345.

11. Trusov A, Bumgarner R, Valijev R, Chestnova R, talevski S, Neeley ES. Comparison of Lumin LED fluorescent attachment, fluorescent microscopy and Ziehl-Neelsen for AFB diagnosis. Int $\mathbf{J}$ Tuberc Lung Dis 2009; 13(7):836-41.
12. Karen R Steingert. Novel approaches and new methods to increase case detection by microscopy 2009;3:120-6.

13. Sohn H, Sinthuwattanawibool C, Rienthong S, Varma JK. Fluorescence microscopy is less expensive than Ziehl-Neelsen microscopy in Thailand. Int $\mathrm{J}$ Tuberc Lung Dis 2009; 13(2):266-268.

14. Jain A, Bhargava A,Agarwal SK. A comparative study of two commonly used staining techniques for acid fast bacilli in clinical specimens. Indian Journal of Tuberculosis 2003; 49:161-2.

15. Githui WA, Matu SW, Muthami LN, Juma E. Improved diagnosis of Ziehl-Neelsen smear negative tuberculosis using sodium hypochlorite sedimentation method. East Afr Med J 2007; 84(10):455-9.

16. Ulukanligil M, Aslan G, Tasci S. A comparative study on the different staining methods and number of specimens for the detection of acid fast bacilli. Mem Inst Oswaldo Cruz 2000; 95(6):855-8.

17. Somoskovi A, Kodman C, LantosA. Comparison of recoveries of Mycobacterium tuberculosis in automated Bactec MGIT 960 TB system and Lowen stein Jensen medium. J Clin. Microbiol 2000; 38(6):2395-2397.

18. Ben J. Marais, Wendy B,Katrien P, Anneke CH. Use of Liggt-Emitting Diode Fluorescence Microscopy to Detect Acid-Fast Bacilli in Sputum. Clinical Infectios Diseases 2008;47:203-7. 